\title{
Accretion mode changes in Centaurus X-3
}

\author{
B. Paul ${ }^{1}$, H. Raichur ${ }^{1,2}$, and U. Mukherjee ${ }^{1}$ \\ 1 Tata Institute of Fundamental Research, Homi Bhabha Road, Colaba, Mumbai 400005, India \\ e-mail: bpaul@tifr.res.in \\ 2 Joint Astronomy Programme, Indian Institute of Science, Bangalore 560012, India
}

Received 19 July 2005 / Accepted 3 September 2005

\begin{abstract}
We report here discovery of the existence of two different accretion modes in the high mass X-ray binary pulsar Cen X-3 during its high states. The multiband X-ray light curves of Cen X-3 lasting for more than 3400 days obtained with the All Sky Monitor (ASM) onboard the Rossi X-ray Timing Explorer (RXTE) shows many episodes of high and low X-ray intensities. The high intensity phases last between a few to upto 110 days and the separation between two high intensity phases also varies widely. One remarkable feature deduced from the RXTE-ASM light curves is that during these high intensity phases, Cen X-3 manifests in two very distinct spectral states. When the source makes a transition from the low intensity phase to the high intensity phase, it adopts one of these two spectral states and during the entire high intensity phase remains in that particular spectral state. During December 2000 to April 2004, all the high intensity episodes showed a hardness ratio which is significantly larger than the same during all the high states prior to and subsequent to this period. It is also found that most of the soft outbursts reach a nearly constant peak flux in the 5-12 keV band. For comparison, similar analysis was carried out on the long term X-ray light curves of three other X-ray binary pulsars Her X-1, Vela X-1, and SMC X-1. Results obtained with these sources are also presented here and we found that none of the other sources show such a behaviour. From these observations, we suggest that Cen X-3 has two different accretion modes and in the course of nine years it has exhibited two switch overs between these.
\end{abstract}

Key words. stars: neutron - pulsars: individual: Cen X-3 - X-rays: stars

\section{Introduction}

The accretion powered X-ray Binary Pulsars (XBP) show $\mathrm{X}$-ray intensity modulations related to the spin of the neutron star, orbital period of the binary, and quasi-periodic oscillations related to motion in the accretion disk. Apart from these, many of the sources also show other short and long time scale intensity variations (Psaltis 2005). Periodic or semi-periodic long term intensity variations are known to be present in a few systems like Her X-1, LMC X-4 and SMC X-1 (Clarkson et al. 2003b; Boyd et al. 2004) which may have a different origin compared to the long term intensity variations in other low and high mass X-ray binaries (Paul et al. 2000; Clarkson et al. 2003b; Still \& Boyd 2004). The quasi-periodic long term intensity variations observed in many X-ray binaries can be due to the precession of a warped inner accretion disk (Shirakawa \& Lai 2002). A stable superorbital period of the warped disk is more common in the high magnetic field neutron star systems (Oglivie \& Dubus 2001). Often the intensity variations at the superorbital period is found to be associated with corresponding complex spectral changes (Naik \& Paul 2002). The spectral changes can be due to variations in the absorption column density in the line of sight and can also have very different fluorescence iron line flux and equivalent width (Naik \& Paul 2004). Long term intensity variations, observed in many other binary systems with low and high mass companion stars are believed to be due to variations in the mass accretion rate caused by changes in the accretion disk characteristics or activity of the companion star (Paul et al. 2000).

Here, we report our investigation of the coarse spectral characteristics of a very well studied High Mass X-ray Binary (HMXB) pulsar Cen X-3 with multi-band light curves taken with the All Sky Monitor (ASM) of the Rossi X-ray Timing Explorer (RXTE) over a long period of 3400 days. A comparison is also made with three other accretion powered $\mathrm{X}$-ray pulsars Her X-1, Vela X-1, and SMC X-1.

\section{Observations, analysis and results}

The RXTE-ASM detectors scan the sky in a series of $90 \mathrm{~s}$ dwells in three energy bands, $1.5-3,3-5$, and 5-12 keV. For details of the instrument and its performance in space, refer to Levine et al. (1996). Here we have used the multi band, perdwell light curves of Cen X-3 and three other bright XBPs Her X-1, Vela X-1, and SMC X-1 provided by the RXTE-ASM team, covering the period of 5 January, 1996 to 12 May, 2005.

The light curves of Cen X-3, in the three different energy bands of ASM, are shown in Fig. 1. As described later, this figure was generated after removing data obtained during the 


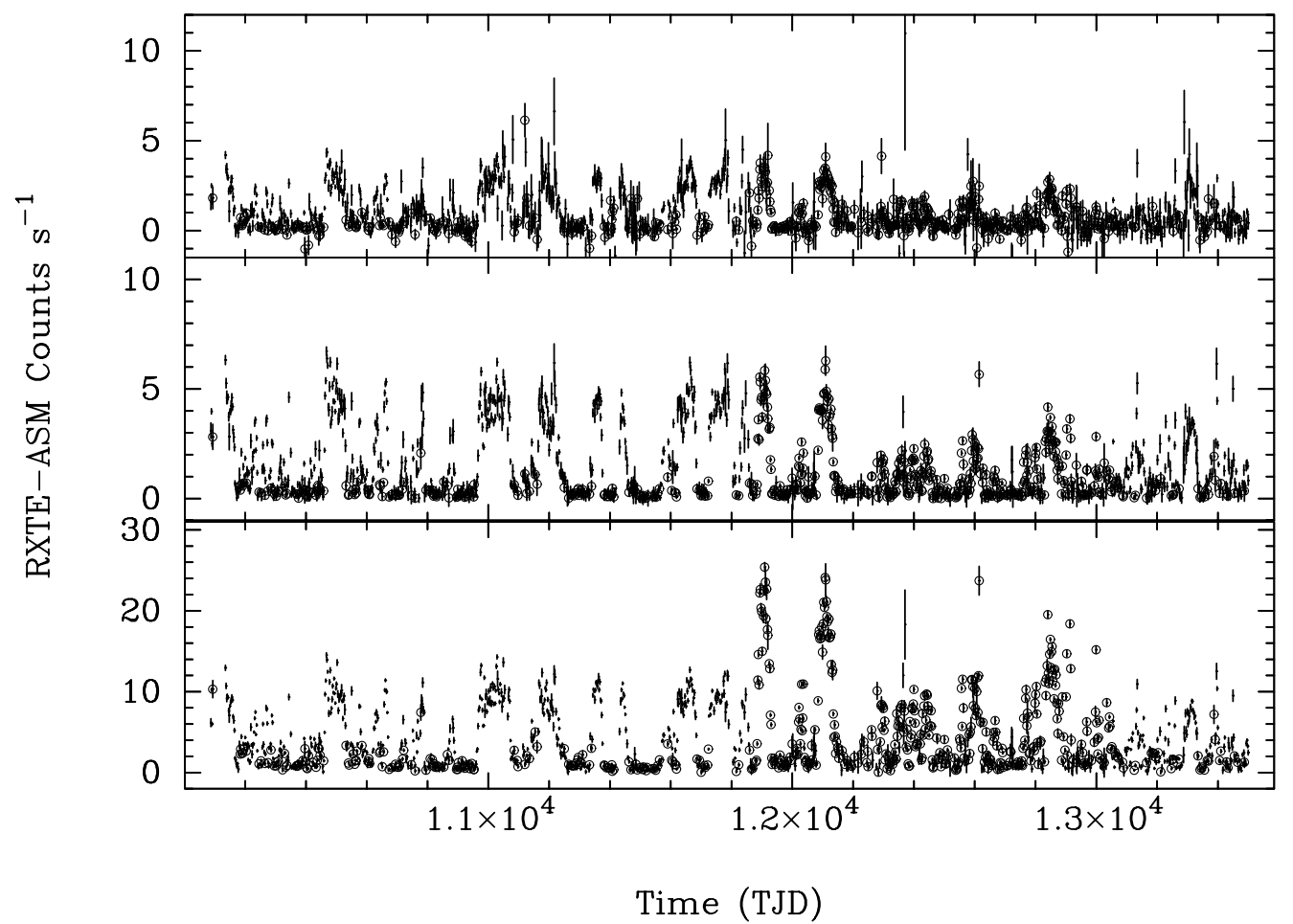

Fig. 1. RXTE-ASM light curves of Cen X-3 in three different energy bands of $1.5-3 \mathrm{keV}, 3-5 \mathrm{keV}$ and 5-12 keV are shown here in top, middle, and bottom panels respectively. Circles are used for data points with hardness ratio (between $5-12 \mathrm{keV}$ and $3-5 \mathrm{keV}$ count rates) greater than 3.5.

X-ray eclipse of Cen X-3 and is binned with a period equal to the orbital period of the binary system. It is clear that the X-ray luminosity of Cen X-3 has a wide variability. The source frequently switches between low and high states with intensity varying by a large factor. In all the three energy bands the peak flux during the bursts is larger compared to the low state flux by upto a factor of 40 . The low and high intensity states last between a few to upto 110 days and occur with no apparent periodicity. The X-ray flux during the low states averaged over a few days is always found to be within about $30 \%$ of the average low state flux during the entire observation period. Period searches with different algorithms did not show any long term periodicity in the intensity variation of Cen X-3. The superorbital intensity variations in two other HMXB pulsars, LMC X-4 (Paul \& Kitamoto 2002) and SMC X-1 (Clarkson et al. 2003a) are smooth in nature, almost sinusoidal over the superorbital period. The other X-ray binary pulsar that shows prominent and stable superorbital intensity variations, when it is out of the occasional anomalous low states is Her X-1 (Still \& Boyd 2004). In this source, each superorbital period consists of one main-on phase and one short-on phase separated by two low intensity phases. In the case of Cen X-3, the rise and fall in intensity around the high states is quite abrupt, lasting about 2-8 days. One interesting feature of the light curve prior to December 2000 (TJD 11880) is that there appears to be a ceiling in the maximum luminosity that the source reaches during the outbursts. The ceiling is most clearly visible in the $5-12 \mathrm{keV}$ band and seems to be present in the $3-5 \mathrm{keV}$ band as well.

For the purpose of this work, we examined the coarse spectral evolution of Cen X-3 as the source went through its intensity changes and calculated two hardness ratios HR1 (3-5 keV/1.5-3 keV) and HR2 (5-12 keV/3-5 keV) from the RXTE-ASM data. A plot of HR1 and HR2 against the ASM count rate is shown in Fig. 2. During the low states, the hardness ratio measurements have large error bars, and in Fig. 2 we have plotted only the data points during which the average ASM count rate is more than 2. The hardness ratio measured during the high states clearly shows a change in spectral shape of the source in the period between December 2000 (TJD 11800) and April 2004 (TJD 13 100). A plot of the medium energy (3-5 keV) X-ray count rate against the hard $\mathrm{X}$-ray $(5-12 \mathrm{keV})$ count rate very clearly shows the existence of two different spectral states in Cen X-3 (Fig. 3). The data points belonging to the two arms in Fig. 3 are from different time ranges in the light curves and are marked in Fig. 1 according to the hardness ratio. Below we describe a careful examination of the various possibilities that may produce such hardness ratio changes artificially. We have ruled out any possibility of the observed spectral changes being an artifact of instrument calibration, sampling etc.

Since this binary system has a small orbital period of 2.09 day, a high mass $\left(\sim 20 M_{\odot}\right)$ companion star, and an inclination angle of $70^{\circ}$ (Ash et al. 1999), it shows a long eclipse lasting for about one fourth of the orbital period during which the X-ray intensity decreases by a large factor. The residual $\mathrm{X}$-ray emission seen during the eclipse is due to the reprocessing of the pulsar X-rays by the stellar wind of the companion star. For individual point X-ray sources, the RXTE-ASM detectors have very short exposures of about $90 \mathrm{~s}$ several times a day. Since eclipse data would contaminate the average X-ray 


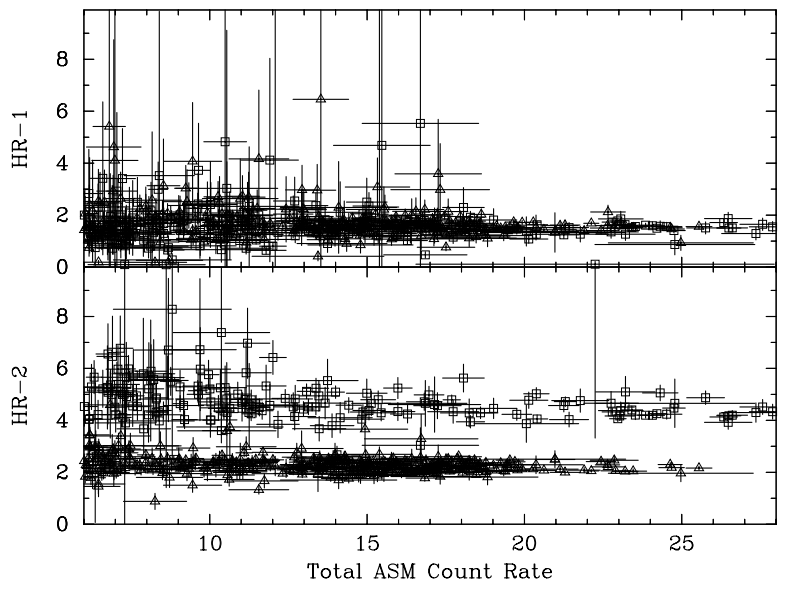

Fig. 2. The two hardness ratios HR-1 and HR-2 derived from the RXTE-ASM light curves of Cen X-3 are plotted here against the ASM count rate for value greater than 2 per second. Data obtained between TJD 11800 and 13100 are shown with square symbol.

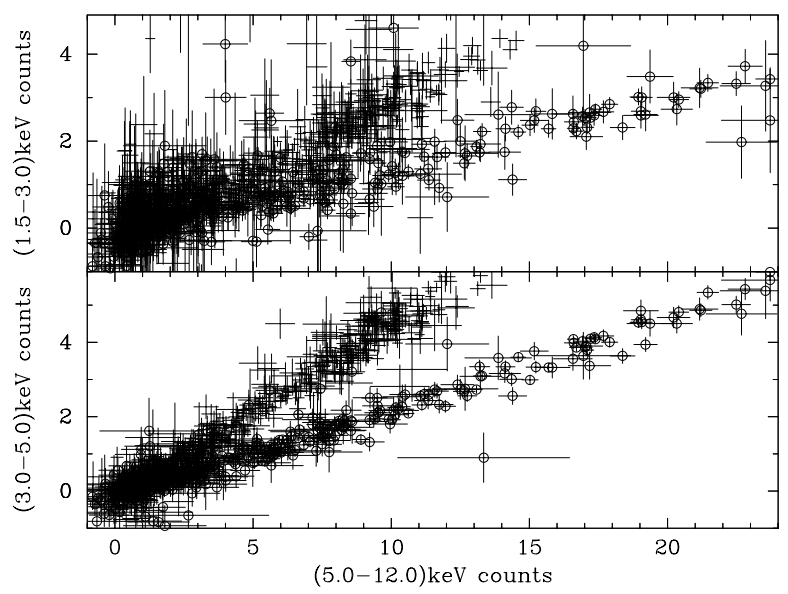

Fig. 3. The RXTE-ASM count rates of Cen $X-3$ in soft $(1.5-3 \mathrm{keV})$ and medium (3-5 keV) energy bands are plotted against the hard $(5-12 \mathrm{keV})$ X-ray count rate. Data obtained between TJD 11800 and 13100 are shown as circles.

flux measurement, all the figures were generated after removing the eclipse data. For all the four sources, the orbital period, mid-eclipse time, and eclipse duration were first derived from the unfiltered RXTE-ASM light curves and are given in Table 1. The orbital periods, derived from the RXTE-ASM data are consistent with the same derived from pulse arrival time analysis. Due to asymmetric nature of the X-ray eclipses, the mid-eclipse times derived from X-ray light curves are not accurate and should not be combined with pulse timing data for study of orbital period evolution. The eclipse durations given in Table 1, include ingress and the egress. To avoid the effect of intensity variations with orbital phase, all the light curves and hardness ratios presented here were produced with a bin size equal to or twice the orbital period.

The RXTE-ASM has three independent detectors with different viewing orientations and makes intensity image of a large part of the sky with a few arc minute angular resolution in one rotation around its axis. Due to this arrangement, the three detectors may obtain different effective exposures for any
Table 1. Orbital parameters of the four XBP sources derived from RXTE-ASM light curves.

\begin{tabular}{llll}
\hline \hline $\begin{array}{l}\text { Source } \\
\text { name }\end{array}$ & $\begin{array}{l}\text { Orbital } \\
\text { period (day) }\end{array}$ & $\begin{array}{l}\text { Mid-eclipse } \\
\text { time (TJD) }\end{array}$ & $\begin{array}{l}\text { Eclipse duration } \\
\text { in orbital phase with } \\
\text { ingress and egress }\end{array}$ \\
\hline Cen X-3 $^{a}$ & $2.08702(3)$ & $10087.295(1)$ & 0.306 \\
Her X-1 $^{b}$ & $1.700165(12)$ & $10088.236(3)$ & 0.318 \\
Vela X-1 $^{c}$ & $3.89201(11)$ & $10087.286(6)$ & 0.231 \\
SMC X-1 $^{d}$ & $8.96461(35)$ & $10087.542(4)$ & 0.377 \\
\hline
\end{tabular}

For orbital parameters of these sources determined from pulse arrival time delays, see ${ }^{a}$ Nagase et al. (1992), ${ }^{b}$ Bhatt et al. (2003), ${ }^{c}$ Deeter et al. (1987), and ${ }^{d}$ Wojdowski et al. (1998).

$\mathrm{X}$-ray source. One of the three detectors is known to have a slightly different energy calibration (A. Levine: private communication). This fact, combined with the varying exposure may also produce some artificial hardness ratio changes in the light curves. To examine this in detail, we have separately analysed data from the three detectors of RXTE-ASM and same result as presented in Figs. 2 and 3 were obtained from all the three detectors.

To ensure that the observed change in spectral hardness ratio for the period December 2000 to April 2004 is not an artifact of changing energy gain calibration of the detectors, we have carried out the same extensive analysis for three other XBP sources Her X-1, Vela X-1 and SMC X-1. As was done with Cen $X-3$, the data acquired during the eclipses were excluded before generating the light curves. Light curves of these three bright XBPs in the broad energy bands of $1.5-12 \mathrm{keV}$ are shown in Fig. 4 with a binsize equal to the respective orbital periods. The duration of the light curves of these three XBPs (Fig. 4) is same as that of Cen X-3 (Fig. 1). In the case of Her X1, data collected during the two anomalous low states (Boyd et al. 2004) were also excluded from analysis. In Fig. 5, the low energy (1.5-3 keV) and medium energy (3-5 keV) X-ray count rates are plotted against the hard X-ray $(5-12 \mathrm{keV})$ count rate for the three sources Her X-1, Vela X-1 and SMC X-1. An integration time twice the orbital period was used for Her X-1 and SMC X-1, while for Vela X-1, it is same as the orbital period. Data obtained between TJD 11800 and 13100 are shown with different symbols. From Fig. 5, one can clearly rule out the possibility that the changes in the hardness ratio detected in Cen X-3 between December 2000 to April 2004 are produced artificially due to any change in energy gain calibration of the detector system. Count rates in the medium $(3-5 \mathrm{keV})$ and hard $\mathrm{X}$-ray $(5-12 \mathrm{keV})$ appear to be very well correlated for Her X-1 and SMC X-1, while for Vela $X-1$ there is significant scatter. We found Cen X-3 to be the only source which had a change in spectral hardness ratio during the period mentioned above.

\section{Discussion}

The continuum energy spectrum of XBP sources is often described by a power-law modified by absorption at low energies, an exponential cutoff at high energies, and cyclotron absorption resonance features (Nagase 1989). Intensity variations in 


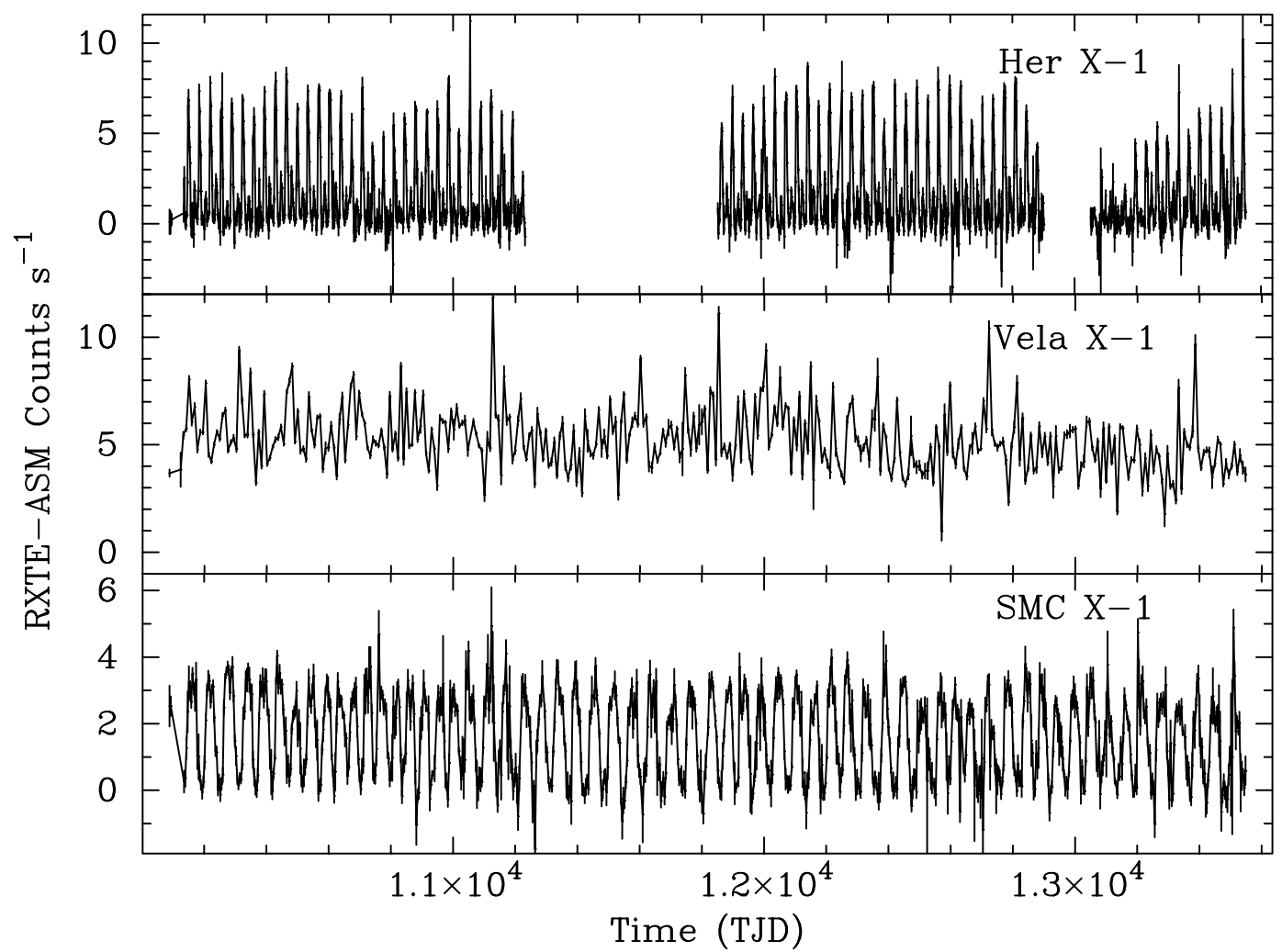

Fig. 4. The 1.5-12 keV RXTE-ASM light curves of Her X-1, Vela X-1, and SMC X-1 are plotted in the top, middle, and bottom panels respectively. For Her X-1, data obtained during the two anomalous low states were omitted.

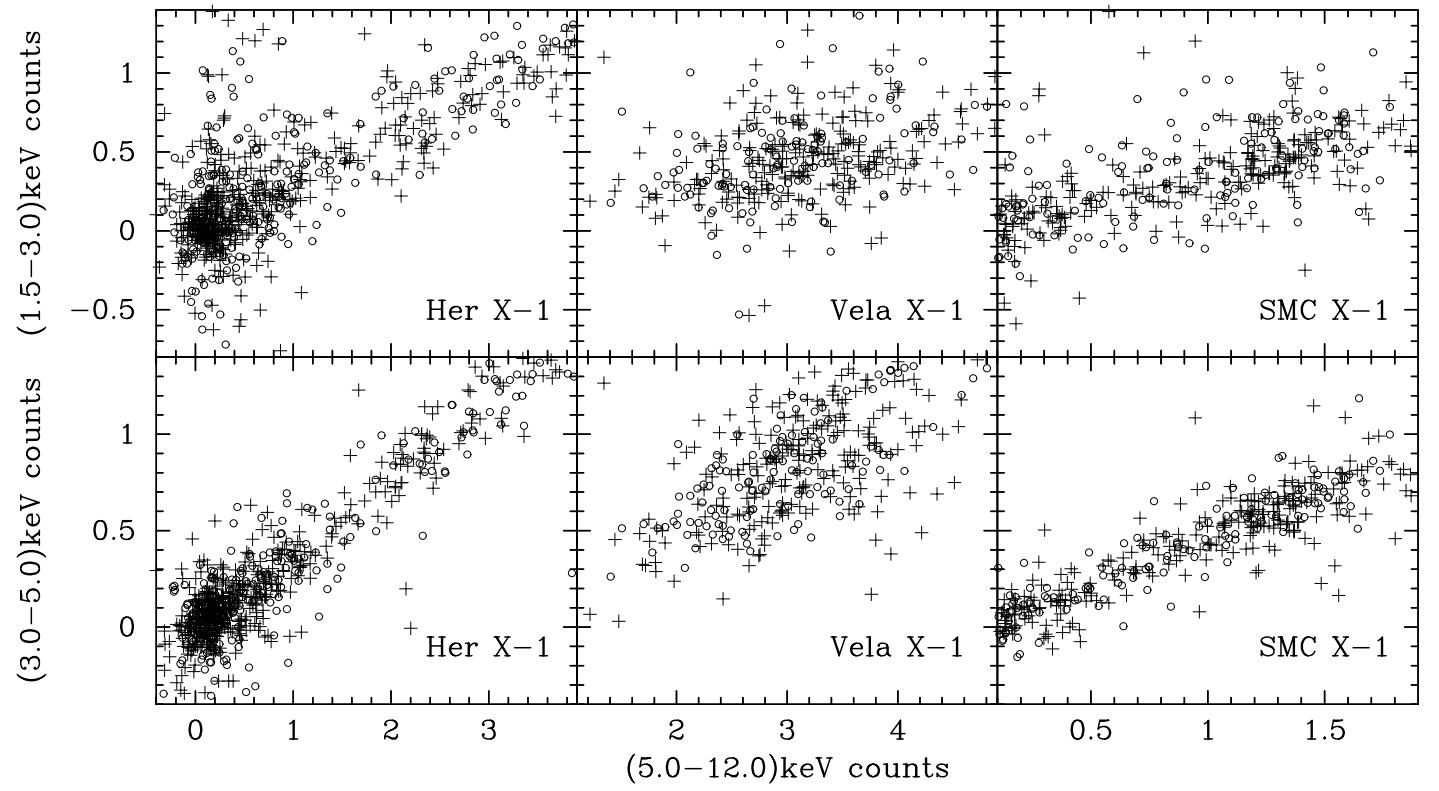

Fig. 5. The RXTE-ASM count rates in soft (1.5-3 keV) and medium (3-5 keV) energy bands are plotted against the hard (5-12 keV) X-ray count rates for the three sources Her X-1, Vela X-1 and SMC X-1. As in Fig. 3, data obtained between TJD 11800 and 13100 are shown as circles. The error bars have not been shown for the sake of clarity.

both transient and persistent XBPs are often associated with changes in the X-ray spectrum and pulse shape, presumably due to modifications in the accretion column near the magnetic poles. Study of the X-ray spectrum in different intensity states is therefore important to understand the reason behind the long term intensity variations.
In the high state, Cen X-3 is the brightest accreting X-ray pulsar and one of the brightest X-ray sources in the energy band of RXTE-ASM. In the present work we have clearly established a few aspects of the long term spectral characteristics of Cen X-3, especially in its high states. In the high state, it has two distinct spectral modes and during each outburst 
the source goes through flux changes via only one of the two spectral modes. During any of the individual high states the source never switches between the two spectral modes. There is no significant spectral evolution during each outburst, at least above a flux level of $100 \mathrm{mCrab}$. All outbursts belonging to one of the two spectral modes have spectral hardness ratio in a very narrow range (Figs. 2 and 3).

Lack of spectral variation during the individual high states is a character of Cen X-3 that is different from sources with precessing warped inner accretion disk. For example, Her X-1 shows a large variation in absorption column density over its 35 day super-orbital period (Naik \& Paul 2003). Presence of the two spectral modes is most clearly seen in the HR-2 (Fig. 2) and is less obvious in HR-1. This indicates that the harder spectrum that occurred during December 2000 to April 2004 is not due to a change in the absorption column density. A change in column density in this period should have had greater effect in the lowest energy band causing a larger difference in the corresponding HR-1 value.

One intriguing feature of Cen X-3 is a ceiling in the maximum luminosity in outbursts prior to the first change in spectral mode that took place around TJD 11880 (Fig. 1, bottom panel). We may speculate that it is related to the Eddington luminosity in Cen X-3 for some limited solid angle in the soft spectral mode.

The pulsed hard X-ray $(20-50 \mathrm{keV})$ flux history of Cen X-3, obtained with CGRO-BATSE shows long term intensity variations identical to variations in the soft $\mathrm{X}$-rays, at least for the period of overlap between CGRO and RXTE observatories (January 1996 to May 2000). However, since the accretion mode with harder spectral shape started after the CGRO era, it is not possible to carry out the corresponding study involving hard X-ray photon flux.

A detailed analysis of the X-ray spectrum in different intensity states of Cen X-3 is in progress and will be reported separately.
Acknowledgements. We thank an anonymous referee for valuable suggestions to improve the paper. We thank the RXTE-ASM and CGRO-BATSE team for providing the valuable data. This research has made use of data obtained from the High Energy Astrophysics Science Archive Research Center (HEASARC), provided by NASA's Goddard Space Flight Center.

\section{References}

Ash, T. D. C., Reynolds, A. P., Roche, P., et al. 1999, MNRAS, 307, 357

Bhatt, N., Paul, B., \& Koul, C. L. 2003, Bull. Astron. Soc. India, 31, 367

Boyd, P. T., \& Smale, A. P. 2004, ApJ, 612, 1006

Clarkson, W. I., Charles, P. A., Coe, M. J., et al. 2003a, MNRAS, 339, 447

Clarkson, W. I., Charles, P. A., Coe, M. J., \& Laycock, S. 2003b, MNRAS, 343, 1213

Deeter, J. E., Boynton, P. E., Lamb, F. K., \& Zylstra, G. 1987, ApJ, 314,634

Levine, A. M., Bradt, H., Cui, W., et al. 1996, ApJ, 469, L33

Nagase, F. 1989, PASP, 41, 1

Nagase, F., Corbet, R. H. D., Day, C. S. R., et al. 1992, ApJ, 396, 147

Naik, S., \& Paul, B. 2002, JApA, 23, 27

Naik, S., \& Paul, B. 2003, A\&A, 401, 265

Naik, S., \& Paul, B. 2004, ApJ, 600, 351

Ogilvie, G. I., \& Dubus, G. 2001, MNRAS, 320, 485

Paul, B., Kitamoto, S., \& Makino, F. 2000, ApJ, 528, 410

Paul, B., \& Kitamoto, S. F. 2002, JApA, 23, 33

Psaltis, D. 2005, in Compact Stellar X-ray Sources, ed. W. H. G. Lewin, \& M. van der Klis (Cambridge: Cambridge Univ. Press) [arXiv: astro-ph/0410536]

Shirakawa, A., \& Lai, D. 2002, ApJ, 565, 1134

Still, M., \& Boyd, P. 2004, ApJ, 606, L135

Wojdowski, P., Clark, G. W., Levine, A. M., Woo, J. W., \& Zhang, S. N. 1998, ApJ, 502, 253 